\title{
Model-Based Factor Analysis of Dynamic Sequences of Cardiac Positron Emission Tomography
}

\author{
R. Klein, Member, IEEE, R.S. Beanlands, R.W. Wassenaar, Member, IEEE, S. Thorn, M. Lamoureux, \\ J.N. DaSilva, A. Adler, R. deKemp, Member, IEEE
}

\begin{abstract}
Factor analysis has been pursued as a means to decompose dynamic cardiac PET images into different tissue types based on their unique temporal signatures. In this work we present a kinetic model-based (MB) method that includes physiological models of factor relationships within the decomposition process. A gamma-variate model was used to describe the transport of ${ }^{82} \mathrm{Rb}$ in arterial blood from the right to left ventricle, and a onecompartment model to describe the exchange between blood and myocardium. Simulations of canine and rat heart imaging were performed to evaluate parameter estimation errors. Arterial blood sampling and ${ }^{11} \mathrm{CO}$ blood pool imaging were used to evaluate factor and structure accuracy. Variable infusion duration studies were used to evaluate MB structure and global myocardial blood flow (MBF) reproducibility. All results were compared with a previously published minimal structure overlap (MSO) method. Simulations demonstrated that MB has lower root-mean-square error (RMSE) for both factor $(\mathbf{p}<0.001)$ and structure $(\mathbf{p}<0.001)$ estimation compared with MSO. Compared to arterial blood samples, MB blood factors had lower RMSE than MSO $(p=0.025)$. There was no difference in the RMSE of blood structures compared with ${ }^{11} \mathrm{CO}$ blood pool images for MB vs. MSO ( $p=0.23$ ). Myocardial structures were more reproducible with MB than with MSO $(\mathrm{p}<0.001)$, as were blood structures $(p=0.006)$. Finally, MBF tended to be more reproducible with MB compared to MSO ( $p=0.16$ ). The execution time of MB was on average 2.4 times shorter than MSO $(p<0.001)$ due to fewer free parameters. Model-based factor analysis can be used to provide physiologically accurate decomposition of ${ }^{82} \mathrm{Rb}$ dynamic PET images.
\end{abstract}

Index Terms-Factor Analysis, Cardiac, PET, 82-Rubidium

Manuscript received June 9, 2009. This work was supported in part by the Natural Sciences and Engineering Research Council (NSERC) Canadian Graduate Scholarship, the Heart and Stroke Foundation of Canada Doctoral Scholarship, Ontario Graduate Scholarship, Canadian Foundation for Innovation Leading Edge Fund Grant \# 11306, Ontario Research Fund grant RE-02-038, Canadian Institute of Health Research Operating Grant \#MOP-79311 and The Heart and Stroke Foundation Molecular Function and Imaging Program Grant \#PRG 6242. *Designate corresponding authors.

*R. Klein is a PhD candidate in Electrical Engineering at the University of Ottawa, Ottawa, Ontario, Canada (e-mail: rklein@ottawaheart.ca).

R. S. Beanlands is Director of the National Cardiac PET Centre at the University of Ottawa Heart Institute, Ottawa, Ontario, Canada.

R. W. Wassenaar is the Physicist of the Nuclear Medicine Department of the Ottawa Hospital, Ottawa, Ontario, Canada.

$\mathrm{S}$. Thorn is a $\mathrm{PhD}$ candidate in Cellular and Molecular Medicine at the University of Ottawa, Ottawa, Ontario, Canada.

M. Lamoureux was a M.Sc. candidate at the Carleton University, Ottawa, Ontario, Canada, Ottawa, Ontario, Canada.

J. N. DaSilva is Head of Radiochemistry at the National Cardiac PET Centre at the University of Ottawa Heart Institute, Ottawa, Ontario, Canada.

A. Adler holds a Canada Research Chair at the Department of Systems and Computer Engineering, Carleton University, Ottawa, Ontario, Canada.

*R. A. deKemp is Head Imaging Physicist at the National Cardiac PET Centre at the University of Ottawa Heart Institute, Ottawa, Ontario, K1Y 4W7, Canada (e-mail: radekemp@ottawaheart.ca).

\section{INTRODUCTION}

$\mathrm{Q}$ uantification of myocardial blood flow (MBF) from dynamic nuclear image sequences has been pursued as a diagnostic and prognostic indicator of heart disease [1]-[3]. Traditionally, an input and output function are derived using image regions of interest. These functions are then used to optimize the parameters of a tracer kinetic model representing a physiologic process of interest such as blood flow. However, the limited spatial resolution and signal-to-noise-ratio of these modalities can limit the accuracy and precision of the imagederived functions [4][5].

Factor analysis techniques have been explored as a means to obtain functions that are free of noise and spillover contamination from adjacent structures [6]. A dynamic series of images is decomposed into a finite number of temporal factors and their corresponding spatial structures which, ideally, should correspond to the physiology of the imaged tissue. The decomposition may be expressed in matrix form as:

$$
\boldsymbol{Y}=\boldsymbol{F S}+\boldsymbol{\varepsilon}
$$

where $\boldsymbol{Y}$ is the dynamic image sequence (the $N$ pixels of each of $M$ time frames in a row), the columns of $\boldsymbol{F}$ contain the timeactivity profiles of the $P$ factors, the rows of $S$ contain the spatial structure of the factor, and $\varepsilon$ is error, or residual signal not accounted for by the factors.

Scaling of factors and structures is arbitrary [7], which is evident from their product in equation 1. Typically the factors are normalized to unity area during decomposition and are later scaled based on the identification of assumed 'pure' pixels in the image [8][9], or based on blood sampling [6]. Even before scaling, decomposition is non-unique [8][10][11], requiring constraints that reduce the range of valid solutions. In cardiac positron emission tomography (PET), these constraints have historically defined non-negative factors and structures [12] based on the physical imaging process. In addition, Poisson statistics have been used to model the imaging process [13], but these constraints still do not ensure a unique solution.

Minimization of the spatial overlap between the structures has been proposed as an additional constraint that ensures uniqueness of the solution. The minimal overlap constraint was originally incorporated as part of a penalized least-squares minimization problem [14][15] and later applied as a successive step[16][17]. Unfortunately, minimal structure overlap partially violates $a$ priori knowledge of structure overlap that exists between blood and myocardium signals due to the limited spatial resolution of PET, and the normal anatomic presence of $10-15 \%$ arterial blood within the myocardial wall. An alternative constraint was 
proposed that minimizes factor overlap under the assumption that the factors should be as distinct as possible temporally [18]. However, there is no absolute physiologic evidence to support this assumption either.

In this work an alternative approach is developed that couples the factors using kinetic models that describe their physiological relationship in time. These models relate the three main components of the cardiac image dynamics - right cavity blood, left cavity blood (including myocardial vasculature), and myocardium (excluding myocardial vasculature). Consequently, model-based factor analysis is specific to this target application and is expected to benefit from increased a priori physiologic information. Cardiac PET with ${ }^{82} \mathrm{Rb}$ may be expected to benefit more than other tracers due to the relatively long positron range, which results in increased image blur, greater mixing of signals and structure overlap. Implementation for ${ }^{82} \mathrm{Rb}$ is relatively simple due to its kinetics being described with a onecompartment model [19], and factor validation is simplified due to lack of blood or tissue metabolites.

In this study we compare the performance of model-based and minimal-spatial overlap constrained factor analyses, using simulation data and experimental data in which arterial blood sampling and ${ }^{11} \mathrm{CO}$ blood pool imaging served as anatomical standards [20]. We also evaluated the reproducibility of structures and MBF under varying image conditions.

\section{METHODS AND MATERIALS}

\section{A. Minimal Structure Overlap (MSO) Constrained Factor Analysis}

MSO decomposition was achieved in two steps: 1) decomposition of the image and 2) rotation of the resulting factors (basis vectors) to minimize spatial overlap as in [16].

Decomposition consisted of minimizing a cost function, $C_{M S O}$, defined as a weighted sum of the norm of the error matrix $|\varepsilon|$, with penalties for negative structure and factor values as expressed by equation 2 , where $W_{E}=1 /|\varepsilon|, W_{N F}=100$ and $W_{N S}=$ 1. Negativity was defined as the norm of all negative elements in matrix $\boldsymbol{X}$ as a ratio of the norm of all elements in $\boldsymbol{X}$ (equations 3 and 4$)$. The same negativity function, $f_{\text {neg }}(\boldsymbol{X}) \in[0,1]$, was used for both factors and structures, substituting $\boldsymbol{F}^{\prime}$ or $\boldsymbol{S}^{\prime}$ for $\boldsymbol{X}$ respectively.

$$
\begin{gathered}
C_{M S O}=W_{E}|\boldsymbol{\varepsilon}|+W_{N F} f_{\text {neg }}\left(\boldsymbol{F}^{\prime}\right)+W_{N S} f_{\text {neg }}\left(\boldsymbol{S}^{\prime}\right) \\
f_{\text {neg }}(\boldsymbol{X})=\frac{1}{|\boldsymbol{X}|} \sqrt{\sum_{i} \sum_{j} H\left(x_{i, j}\right)} \\
H(x)=\left\{\begin{array}{cl}
x^{2}, & x<\epsilon \\
0, & x \geq \epsilon
\end{array}\right.
\end{gathered}
$$

The cost function, $C_{M S O}$, was minimized by optimizing $\boldsymbol{F}$, using a simplex gradient descent algorithm (fminsearch, Matlab, The MathWorks, Boston, MA) that was executed in several iterations (epochs) until the variance of $\varepsilon$ stabilized to a value less than $10^{-6}$ of the mean. In each epoch the error penalty, $W_{E}$, was set to the value of $1 /|\varepsilon|$ from the previous epoch. This approach iteratively estimated the amount of noise in the image, resulting in consistent relative penalty weights without the need to assume a particular noise level.
In the second step, the MSO constraint was applied to minimize the weighted sum of factor and structure negativity penalties as well as spatial overlap penalty, $f_{\text {ovl }}(\boldsymbol{X}) \in[0,1]$, as shown in equations 5 and 6 . The matrices $F^{\prime}$ and $S^{\prime}$ are the factor and structure matrices resulting from the decomposition stage and $\boldsymbol{R}$ is a $P \times P$ rotation matrix that is optimized to minimize $f_{\text {tot }}{ }^{M S O}$, where $P$ in the number of factors. The resulting factors and structures were defined as $\boldsymbol{F}=\boldsymbol{F}^{\prime} \boldsymbol{R}$ and $\boldsymbol{S}=\boldsymbol{R}^{-1} \boldsymbol{S}^{\prime}$ respectively. The parameter $b(=0.001)$ was used to weight the overlap penalty, giving priority to the non-negativity constraint.

$$
\begin{gathered}
f_{\text {tot }}^{M S O}=f_{\text {neg }}\left(\boldsymbol{F}^{\prime} \boldsymbol{R}\right)+f_{\text {neg }}\left(\boldsymbol{R}^{-1} \boldsymbol{S}^{\prime}\right)+b f_{\text {ovl }}\left(\boldsymbol{R}^{-1}, \boldsymbol{S}^{\prime}\right) \\
f_{\text {ovl }}(\boldsymbol{X})=\frac{2}{P(P-1)} \sum_{p=1}^{P} \sum_{q=p+1}^{P} \sum_{i=1}^{N} \frac{\left|X_{i p}\right|}{\sqrt{\sum_{j=1}^{N} x_{j p}^{2}}} \frac{\left|X_{i q}\right|}{\sqrt{\sum_{j=1}^{N} x_{j q}^{2}}}
\end{gathered}
$$

\section{B. Model-Based Factor Analysis (MB)}

The right ventricle (RV) cavity blood factor was treated as an input function that could take any shape, with unit area. Thus the RV factor, $r(t)$, was considered a free variable vector with $M-1$ degrees of freedom where $M$ is the number of time frames. The left ventricle (LV) cavity blood was modeled by convolution of the RV blood with a model of the delay and dispersion resulting from transport of the RV blood through the lungs and pulmonary vessels to the LV. We adopted a standard gamma-variate function (equation 7) to model this transport [20]-[24], where the model parameter $t_{p}$ represents time delay to peak activity [21], and $\beta$ controls the broadness of dispersion.

$$
G(t)=t^{\beta} e^{-\beta t / t_{p}}
$$

The myocardium factor was modeled by convolution of the LV blood factor with a compartmental response function assumed for the specific tracer. In the case of ${ }^{82} \mathrm{Rb}$, a one compartment model impulse response function was used [19] with a single free parameter $k_{2}$ (tracer washout) as in equation 8 .

$$
M(t)=e^{-k_{2} t}
$$

Since the factors were scaled to unit area as part of the decomposition process, the scaling factors typically included in equations 7 and 8 are redundant, and so were removed. The use of these models to parameterize two of the three factors reduces the number of free parameters from $3(M-1)$ with MSO to $M+2$ with the MB method. In addition, coupling of the factors through the modeled response functions imposes a constraint on the solution that agrees with our knowledge of the tracer physiology.

\section{1) Optimization}

The factor matrix, $\boldsymbol{F}$, was constructed of columns containing RV blood, LV blood, and myocardium factors (equation 9), where the symbol $\otimes$ denotes the discrete point convolution operation and integration over the image time frames.

$$
\boldsymbol{F}=\left[\begin{array}{c}
r(t) \\
r(t) \otimes G \\
r(t) \otimes G \otimes M
\end{array}\right]^{T}
$$


The model-based decomposition process consisted of optimization of the RV blood factor simultaneously with the model parameters to minimize the objective function, $C_{M B}$, (equation 10) which contains the same error and non-negativity penalties used in MSO, but does not include a penalty for spatial overlap. To ensure uniqueness and robustness of the MB solution two penalties were added, $f_{B R}$ and $f_{R}$ :

$f_{B R} \in[0,1]$ - Penalizes for blood factors that increase in the uptake phase. This penalty was defined as the area bound by the activity of the last RV blood factor time frame, $\boldsymbol{F}_{1, M}$, and activity of time frames post peak RV blood that are below $\boldsymbol{F}_{1, M}$, as demonstrated by the light shaded area in Fig. 1. This penalty aids convergence by promoting solutions with monotonic decrease in blood activity past the blood peak activity, again consistent with the known physiology.

$f_{R} \in[0,1]$ - Penalizes for residual blood activity in the uptake phase (past the first 2 minutes) as demonstrated by the dark shaded area in Fig. 1.

$$
C_{M B}=W_{E}|\boldsymbol{\varepsilon}|+W_{N F} f_{n e g}(\boldsymbol{F})+W_{N S} f_{n e g}(\boldsymbol{S})+W_{B R} f_{B R}+W_{R} f_{R}
$$

The cost function, $C_{M B}$, was optimized using the same routine used to optimize $C_{M S O}$. The penalty weights $W_{B R}$ and $W_{R}$ were empirically set to 10 and 0.01 respectively, while $W_{E}, W_{N F}$, and $W_{N S}$ were maintained the same as for MSO. The model parameters were bound based on typical experimental values (Table I). Thus, while MSO solves the ambiguity problem by minimizing the overlap between structure, MB imposes a priori physiologic constraints on the shape of the blood factor, as well as the other factors via the kinetic models.

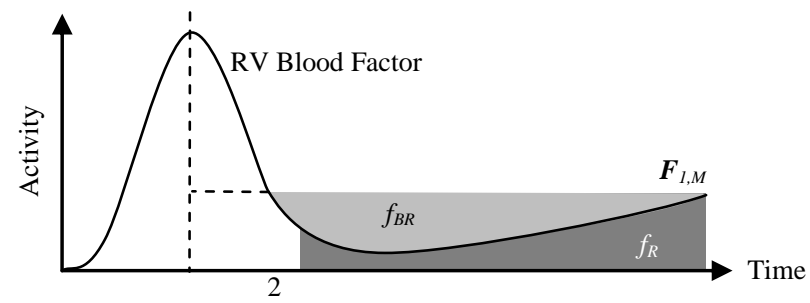

Fig. 1 - Blood increase penalty, $f_{B R}$, is the integral of RV blood that is beyond the initial peak and below activity of the last time frame (light shaded area). Residual blood activity, $f_{R}$, is the area under the curve from 2 minute to the end of the scan (dark shaded area).

\section{Number of Factors}

For both MSO and MB methods, the number of factors ( 2 or 3 in this case) was determined automatically as the value beyond which $|\varepsilon|$ decreased by less than $1 \%$. In the event of decomposition into only two factors, a single blood factor and a myocardium factor were always resolved. In this case the blood factor was the free variable and the myocardium was modeled

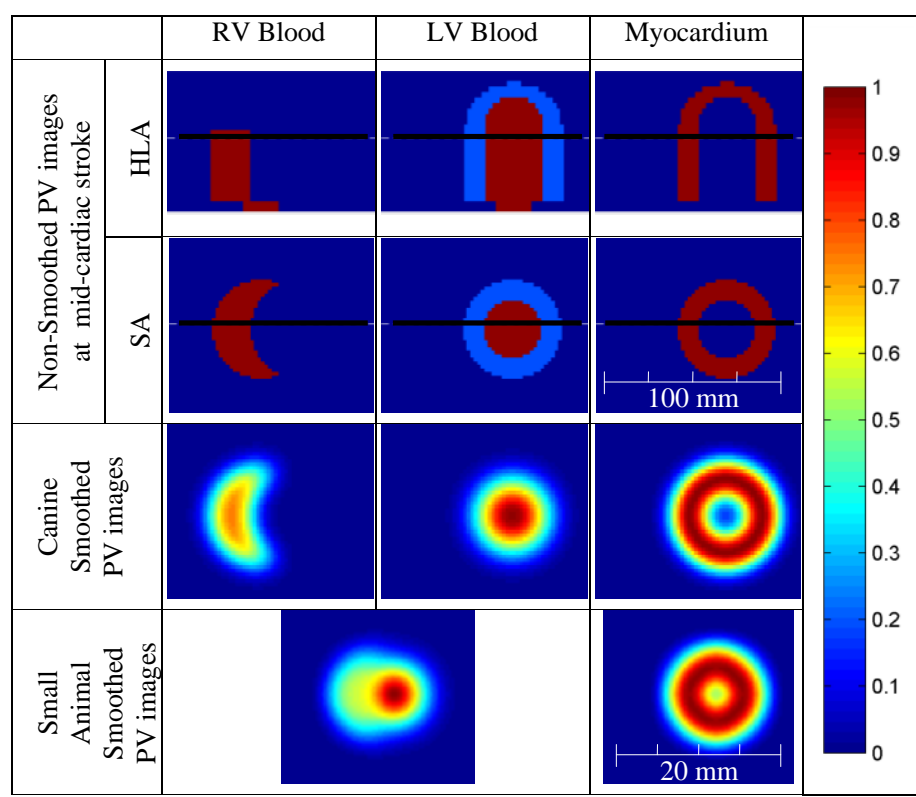

Fig. 2 - Horizontal long axis (top row) and short axis slices (second row) of simulated structures with a black line showing their intersection. The shortaxis slices of the smoothed structures simulate canine images (third row) and small animal images (bottom row). All images are scaled to maximum intensity pixel to emphasize contrast.

using the compartmental model (equation 8).

\section{Simulations}

\section{1) Canine Simulation}

RV blood time activity curves were simulated as a $30 \mathrm{sec}$, constant rate of activity infusion with dispersion (modeled with a gamma-variate function) en route to the RV cavity. Two forms of $\mathrm{RV}$ blood TAC were created to assess the solution's robustness to different input function characteristics:

- Complete clearance of blood activity

- $\quad$ Residual (non-zero) blood activity

Each RV blood TAC was convolved with a gamma-variate impulse response function (equation 7) to generate an LV factor. The LV factor in turn was convolved with a one-compartment model impulse response function (equation 8) to generate a myocardium factor. The model parameters used for the simulations are shown in Table I. Different washout parameters, $k_{2}$, were selected for the complete blood clearance and residual blood activity cases so that the myocardial TAC had a similar appearance during the late uptake phase of the dynamic image sequence. Each factor was sampled by integrating over 17 time intervals as used in clinical practice $(12 \times 10 \mathrm{~s}, 2 \times 30 \mathrm{~s}, 1 \times 60 \mathrm{~s}$, $1 \times 120$ s, and $1 \times 240$ s).

These factors were then cross multiplied with their respective partial volume (PV) images representing the anatomic structures

TABLE I

ERROR IN RESOLVED PHYSIOLOGIC PARAMETER VALUES WITH MODEL-BASED ANALYSIS (\%)

\begin{tabular}{|c|c|c|c|c|c|c|c|c|c|c|}
\hline \multirow{2}{*}{$\begin{array}{l}\text { Blood } \\
\text { Clearance }\end{array}$} & \multirow{2}{*}{$\begin{array}{l}\text { Parameter } \\
\text { Name }\end{array}$} & \multirow[t]{2}{*}{ units } & \multirow{2}{*}{$\begin{array}{l}\text { Lower } \\
\text { Bound }\end{array}$} & \multirow{2}{*}{$\begin{array}{l}\text { Initial } \\
\text { Estimate }\end{array}$} & \multirow{2}{*}{$\begin{array}{l}\text { Upper } \\
\text { Bound }\end{array}$} & \multirow{2}{*}{\begin{tabular}{|l} 
Simulated \\
Value
\end{tabular}} & \multicolumn{2}{|c|}{ Canine Simulation } & \multicolumn{2}{|c|}{ Small Animal Simulation } \\
\hline & & & & & & & No Noise & $10 \%$ Noise & No Noise & $10 \%$ Noise \\
\hline \multirow{3}{*}{ Complete } & $\mathrm{t}_{\mathrm{p}}$ & $\sec$ & 2 & 10 & 30 & 15.0 & 15.0 & $15.2 \pm 0.5$ & - & - \\
\hline & $\beta$ & - & 0.25 & 1 & 5 & 2.00 & 2.00 & $1.95 \pm 0.09$ & - & - \\
\hline & $\mathrm{k}_{2}$ & $\min ^{-1}$ & 0.01 & 0.1 & 1.5 & 0.050 & 0.050 & $0.051 \pm 0.004$ & 0.050 & $0.049 \pm 0.001$ \\
\hline \multirow{3}{*}{ Residual } & $\mathrm{t}_{\mathrm{p}}$ & $\sec$ & 2 & 10 & 30 & 15.0 & 15.0 & $15.6 \pm 0.6$ & - & - \\
\hline & $\beta$ & - & 0.25 & 1 & 5 & 2.00 & 2.00 & $2.24 \pm 0.27$ & - & - \\
\hline & $\mathrm{k}_{2}$ & $\min ^{-1}$ & 0.01 & 0.1 & 1.5 & 0.500 & 0.500 & $0.496 \pm 0.036$ & 0.050 & $0.499 \pm 0.037$ \\
\hline
\end{tabular}


(dimensions shown in Table II). Representative horizontal long axis (HLA) and short axis (SA) images are shown in Fig. 2. Each PV image of the simulation was averaged over 36 gates of sinusoidal cardiac motion and wall thickening to preserve the myocardial mass across all gates. Of the dynamic image sequences, one image did not have noise added to it while 5 images had Gaussian distributed random noise added. The images were then smoothed using a 13.6 mm FWHM Gaussian filter. The variance of the noise in each pixel was proportional to the pixel intensity [25]. The noise gain was adjusted (100\%) to generate the same postsmoothing image variance in the myocardial region as measured in our canine images with our clinical scanner $(\sim 10 \%$ variance). By adding noise in image-space we assumed that scatter and attenuation were properly corrected for in the experimental data. Short axis slices of the canine motion blurred and spatially smoothed PV images are shown in Fig. 2.

\section{2) Small Animal Simulation}

Small animal images were simulated in a similar manner as the canine studies, but the dimension were changed as shown in Table II and the smoothing kernel was set to $4.4 \mathrm{~mm}$ FWHM, corresponding roughly to ${ }^{82} \mathrm{Rb}$ imaging with a small animal PET system. Since RV and LV blood could not be distinguished in small animal ${ }^{82} \mathrm{Rb}$ PET images, a single blood component was used. The blood structure was created by summation of the $\mathrm{LV}$ and RV blood structures. The resulting, motion blurred and smoothed, PV images are shown in Fig. 2. The same LV blood and myocardium TACs from the canine simulation were used for the small animal simulation.

TABLE II

SIMULATED IMAGE DiMENSIONS

\begin{tabular}{|l|r|r|}
\hline Parameter & Canine & Small Animal \\
\hline Short axis slice pixel size $(\mathrm{mm})$ & 1.72 & 0.345 \\
\hline Short axis slice thickness $(\mathrm{mm})$ & 2.62 & 0.80 \\
\hline LV diameter (end systole) $(\mathrm{mm})$ & 50.40 & 10.67 \\
\hline Wall thickness (mid cycle) $(\mathrm{mm})$ & 7.00 & 1.00 \\
\hline LV wall motion $(\mathrm{mm})$ & 12.60 & 2.67 \\
\hline RV cavity width (end systole) $(\mathrm{mm})$ & 20.59 & 2.76 \\
\hline
\end{tabular}

\section{3) Analysis of Simulation Studies}

The canine and small animal simulated images were decomposed using both MSO and MB. Factors and structures were compared against the respective TACs and PV images from the simulation, measuring percent root-mean-squarederror (RMSE\%) as shown in equation 11 where $\boldsymbol{X}_{\boldsymbol{r e f}}$ is a reference matrix from the simulation and $\boldsymbol{X}$ is the results matrix that is being evaluated. For factor evaluation, the weighting matrix, $\boldsymbol{W}$, was the length of the imaging time frames, and for structure validation $\boldsymbol{W}$ was all ones, weighting each pixel equally. As shown in equation 11, the matrices $\boldsymbol{X}, \boldsymbol{X}_{\boldsymbol{r e f}}$, and $\boldsymbol{W}$ were each normalized by their sum.

$$
R M S E \%=\left|\frac{\boldsymbol{W}}{\sum \boldsymbol{W}} \cdot\left(\frac{\boldsymbol{X}}{\sum \boldsymbol{X}}-\frac{\boldsymbol{X}_{\text {ref }}}{\sum \boldsymbol{X}_{\text {ref }}}\right)\right| \times 100 \%
$$

Contrast in the myocardium structures was compared with that in the smoothed PV images. Contrast was defined using equation 12, where $M y o$ is the maximal intensity in the

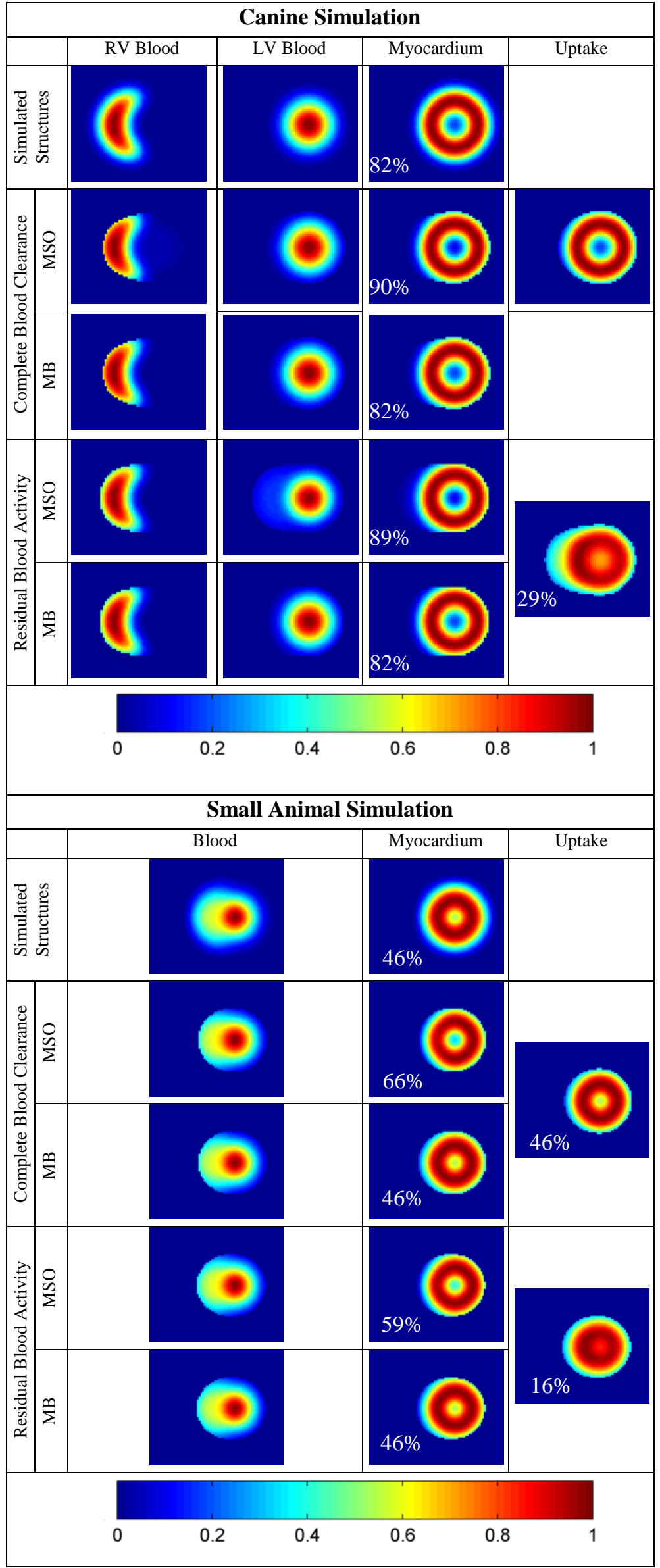

Fig. 3 - Short axis slices of decomposed structures using MSO and MB methods on canine (top half) and small animal (bottom half) simulated dynamic images are compared with simulated structures and uptake images (frame 17). White numbers are contrast between myocardium and spillover into the LV cavity (equation 12). 
myocardium structure and $\mathrm{Cav}$ is the mean intensity of the four pixels in the center of the cavity of the SA slice shown in Fig. 2.

$$
\text { Contrast }=\frac{M y o-C a v}{M y o} \times 100 \%
$$

Robustness of convergence of the MB algorithm was assessed by modifying the initial model parameter estimates (default values in Table I) to $t_{p}=5 \& 20 \mathrm{sec}, \beta=0.5 \& 3$, and $k_{2}=0.01 \& 1$ $\mathrm{min}^{-1}$ and decomposing the simulated canine images (resulting in 7 sets of decomposed images). The resulting $k_{2}$ parameter estimates were analyzed using box plots for changes in means and/or variances as a result of modifying the initial parameters.

The resolved model parameters from MB were compared to the simulated model parameters. Since, MSO does not resolve similar parameters, an estimate of the washout parameter, $k_{2}$, was calculated for both MSO and MB by fitting the one compartment model (equation 13) to the LV blood and myocardium factors $(l(t)$ and $m(t)$ respectively). The parameter $T B V$ represents the total blood volume in the ROI and $K_{l}$ is the uptake rate, but both these parameters were not analyzed.

$$
m(t)=T B V \cdot l(t)+(1-T B V) \cdot K_{1} l(t) \otimes e^{-k_{2} / t}
$$

\section{E. Experimental Studies}

All animal experiments were conducted in accordance with protocols approved by the Animal Care Committee of the University of Ottawa.

\section{1) Factor Validation with Arterial Blood Sampling}

To test the physiological accuracy of factors (temporal domain), blood factors were compared with activity concentration measurements in arterial blood. Four rats were imaged using ${ }^{82} \mathrm{Rb}$ a total of 6 times with simultaneous and continuous blood sampling. Rats were anesthetized with 1.5-2\% isoflurane and a PE50 catheter was surgically introduced into the carotid artery and fed into the left atrium. A catheter was inserted into the tail vein for tracer injection and a 3-lead ECG, rectal thermometer, and respiration monitor were affixed for physiologic monitoring. A heated bed was used to maintain normal body temperature throughout the experiment.

The rat was placed in an Inveon DPET (Siemens, Knoxville, $\mathrm{TN})$ scanner with the heart centered in the field of view. The arterial catheter was attached to a micro-volumetric blood activity counter [26] (AMI, Sherbrooke, Quebec) for 2-3ml of blood withdrawal over a 10 min time interval. The PET scan and blood counter were started together approximately 10 seconds before manual injection of ${ }^{82} \mathrm{Rb}$ from a $1 \mathrm{ml}$ syringe, and followed by a $0.4 \mathrm{ml}$ saline flush to clear activity from the catheter lock. List-mode data were acquired for 10 min using a $350-650 \mathrm{keV}$ energy window and a coincidence timing window of $3.4 \mathrm{~ns}$. The dynamic data (same time frames as simulation) were reconstructed on a $128 \times 128$ image matrix with $0.345 \times 0.345 \times 0.80 \mathrm{~mm}$ pixel size using OSEM $3 \mathrm{D} / \mathrm{MAP}(\beta=1.0$, OSEM3D iterations=2, MAP iterations $=18$ ) with corrections for dead-time, isotope decay, detector efficiencies, and randoms. The image sequences were decomposed using both MSO and MB methods as described above.

Since arterial blood sampled time-activity concentration curves could not be corrected for background activity after tracer injection using the vendor provided software [26], we implemented our own analysis of the raw counts. Counts were corrected for tracer decay, background activity (10 sec preceding first rise in sampled activity), delivery delay, and the spatial resolution of the $\beta^{+}$detector. In addition, the corrected blood curves were integrated over the same time intervals as the dynamic image sequence. The integrated curves were normalized to unit area before comparison with the MSO and MB derived blood factors using RMSE (\%) with each time frames weighed by its length normalized by the total scan duration as in equation 11 .

\section{2) Structure Validation with ${ }^{11}$ CO Blood Pool Imaging}

A single dog was anesthetized using $~ 2 \%$ isoflurane and positioned in a whole-body PET scanner (ECAT ART, Siemens/CTI, Knoxville, TN) with the heart centered in the fieldof-view. A series of ten dynamic scans (same time frames as simulation) was acquired with varying ${ }^{82} \mathrm{Rb}$ constant-activity (150 MBq) rate infusion [27] durations $(15,30,60,120,240$, $240,120,60,30,15$ seconds). The images were reconstructed iteratively (OSEM) to $12 \mathrm{~mm}$ resolution.

The images were cropped manually to include the entire heart (same crop applied to all 10 images). The cropped regions were then decomposed using both MSO and MB methods. The number of factors was automatically determined, but in cases having two blood structures (RV and LV), they were summed to form a single combined blood pool structure.

The blood structures extracted from each of the 10 images were compared to a single ${ }^{11} \mathrm{CO}$ blood pool image of the same animal acquired on a separate day and reconstructed to the same $12 \mathrm{~mm}$ resolution. ${ }^{11} \mathrm{CO}$ binds to hemoglobin in the blood resulting in images of the total blood distribution, therefore serving as a good anatomic reference to validate the accuracy of the factor analysis blood structures.

Contrast in the myocardium structures was compared with that of the uptake phase (last 6 minutes) images. Contrast was defined using equation 12, where $M y o$ and $C a v$ are the mean pixel intensities in the LV myocardium and LV blood cavity ROIs respectively. The ROIs were automatically determined [19][28].

\section{3) Global MBF Reproducibility using Variable Tracer Infusion Durations}

Global MBF was quantified in the same images. The myocardium ROI was used to sample kinetic modeling output function, $m(t)$, from the dynamic image sequences. Likewise, the $\mathrm{LV}$ blood cavity ROI $\left(\mathrm{ROI}_{\mathrm{b}}\right)$ was used to sample the kinetic model input function, $l(t)$. These were used with the kinetic model of equation 13 to determine uptake rate, $K_{l}$, and MBF was calculated from $K_{I}$ using a previously derived Renkin-Crone extraction function [19].

The MSO and MB blood factors were used as alternative input functions in equation 13 and were scaled by the average blood structure in the $\mathrm{ROI}_{\mathrm{b}}$. If two blood factors (RV and LV) existed they were both scaled using their respective structure $\mathrm{ROI}_{\mathrm{b}}$ and summed, thus accommodating incomplete spatial separation of the blood factors into RV and LV cavity blood. We also evaluated the benefit of including the RV blood time activity curve, $r(t)$, in the kinetic model using equation 14 where RBV and LBV are the right and left blood PV contributions in the myocardium ROI. The maximum pixel value in the RV structure was used to scale $r(t)$. 
TABLE III

Simulated Image ReSults - RoOT MeAn SquAREd ERRoR (\%)

\begin{tabular}{|c|c|c|c|c|c|c|c|c|c|c|c|}
\hline \multicolumn{12}{|c|}{ Canine Simulated Data } \\
\hline \multirow{3}{*}{$\begin{array}{r}\text { Blood } \\
\text { Clearance }\end{array}$} & \multirow{3}{*}{ Factor } & \multicolumn{5}{|c|}{ Factors } & \multicolumn{5}{|c|}{ Structures } \\
\hline & & \multicolumn{2}{|c|}{ No Noise } & \multicolumn{3}{|c|}{$10 \%$ Noise $(\mathrm{n}=5)$} & \multicolumn{2}{|c|}{ No Noise } & \multicolumn{3}{|c|}{$10 \%$ Noise $(\mathrm{n}=5)$} \\
\hline & & MSO & $\mathrm{MB}$ & MSO & MB & $\mathrm{p}$ & MSO & MB & MSO & $\mathrm{MB}$ & $\mathrm{P}$ \\
\hline \multirow{3}{*}{ Complete } & RV & 0.0 & 0.0 & $0.3 \pm 0.1$ & $0.3 \pm 0.2$ & 1.000 & 1.4 & 0.0 & $2.7 \pm 0.5$ & $1.7 \pm 0.1$ & 0.015 \\
\hline & LV & 0.6 & 0.0 & $0.6 \pm 0.1$ & $0.1 \pm 0.0$ & 0.001 & 1.3 & 0.0 & $2.9 \pm 1.2$ & $2.7 \pm 0.6$ & 0.658 \\
\hline & Myo & 0.2 & 0.0 & $0.3 \pm 0.1$ & $0.1 \pm 0.1$ & 0.001 & 2.2 & 0.0 & $4.2 \pm 1.0$ & $3.8 \pm 0.7$ & 0.059 \\
\hline \multirow{3}{*}{ Residual } & RV & 1.3 & 0.0 & $0.9 \pm 0.4$ & $0.2 \pm 0.1$ & 0.027 & 0.0 & 0.0 & $2.7 \pm 0.3$ & $1.6 \pm 0.1$ & 0.002 \\
\hline & LV & 0.2 & 0.0 & $0.5 \pm 0.2$ & $0.2 \pm 0.1$ & 0.095 & 7.1 & 0.0 & $8.0 \pm 1.8$ & $2.8 \pm 0.6$ & 0.001 \\
\hline & Myo & 0.5 & 0.0 & $0.7 \pm 0.2$ & $0.1 \pm 0.0$ & 0.004 & 2.9 & 0.0 & $7.5 \pm 0.8$ & $5.6 \pm 1.3$ & 0.0275 \\
\hline \multicolumn{2}{|l|}{ Mean } & 0.5 & 0.0 & $0.5 \pm 0.3$ & $0.2 \pm 0.1$ & $<0.001$ & 2.5 & 0.0 & $4.7 \pm 2.5$ & $3.0 \pm 1.5$ & $<00.001$ \\
\hline \multicolumn{12}{|c|}{ Rat Simulated Data } \\
\hline \multirow{2}{*}{ Complete } & Blood & 1.7 & 0.0 & $1.7 \pm 0.2$ & $0.2 \pm 0.1$ & $<\mathbf{0 . 0 0 1}$ & 1.8 & 0.0 & $6.0 \pm 2.9$ & $1.7 \pm 0.3$ & $<0.001$ \\
\hline & Myo & 0.2 & 0.0 & $0.7 \pm 0.3$ & $0.2 \pm 0.1$ & 0.023 & 6.2 & 0.0 & $6.7 \pm 0.8$ & $2.5 \pm 0.6$ & $<0.001$ \\
\hline \multirow{2}{*}{ Residual } & Blood & 0.4 & 0.0 & $0.4 \pm 0.1$ & $0.4 \pm 0.2$ & 0.529 & 0.6 & 0.0 & $8.4 \pm 2.2$ & $1.9 \pm 0.6$ & $<0.001$ \\
\hline & Myo & 0.1 & 0.0 & $0.8 \pm 0.1$ & $0.1 \pm 0.0$ & $<0.001$ & 4.1 & 0.0 & $5.9 \pm 1.1$ & $5.9 \pm 2.2$ & $<0.001$ \\
\hline \multicolumn{2}{|l|}{ Mean } & 0.6 & 0.0 & $0.9 \pm 0.5$ & $0.2 \pm 0.1$ & $<0.001$ & 3.2 & 0.0 & $6.7 \pm 2.1$ & $3.0 \pm 2.1$ & $<0.001$ \\
\hline
\end{tabular}

$$
\begin{aligned}
m(t)= & R B V \cdot r(t)+L B V \cdot l(t) \\
& +(1-R B V-L B V) \cdot K_{1} e^{-k_{2} t} \otimes l(t)
\end{aligned}
$$

Reproducibility of MBF using the ROI based method as well as using all combinations of MB and MSO, with and without RV blood were evaluated.

\section{4) Structure Reproducibility using Variable Tracer Infusion Durations}

The myocardium and blood structures obtained from the 10 variable infusion duration images were compared (RMSE\%) in all possible combinations $(n=45)$ to determine the reproducibility of blood and myocardium structures. In addition the coefficient of variation of each pixel across the 10 images was computed and was then averaged across all the pixels in the regions of interest, and was referred to as $\mathrm{CV} \%$. CV\% was measured for blood structures and myocardium structures both for MSO and MB.

\section{F. Statistical Analysis}

Unless otherwise specified, all values are reported as mean \pm standard-deviation. Comparison of means was performed using a two-tailed paired student t-tests, with $\mathrm{p}=0.05$ as the cutoff for significance. Comparison of variability was performed using the parametric f-tests with the same significance cutoff. Both the student t-test and the f-test assume normal Gaussian distribution, which was not explicitly tested. Statistics regarding images and/or structures relate to all pixels within the entire region of interest.

Comparison of population variables is presented using notched box plots showing the median and inter-quartile range. Outliers are identified outside the whiskers extending to 1.5 times the quartiles. Non-overlapping notches reflect significantly different medians with $95 \%$ confidence.

The Wilcoxon, non-parametric rank sum test was used to test the significance of differences in $\mathrm{CV} \%$ with $\mathrm{p}=0.05$ as the cutoff.

\section{RESULTS}

\section{A. Canine Simulation}

Table III lists the RMSE between the simulated and resolved factors and structures using both decomposition methods. With few exceptions, MB errors were lower than corresponding MSO errors. With no noise the mean factor RMSE was $0.5 \%$ with $\mathrm{MSO}$, while all factor RMSEs were $<0.05$ with MB. With noise the MSO factors had significantly $(\mathrm{p}<0.001)$ higher RMSE values $(0.5 \%)$ compared to MB $(0.2 \%)$. Similarly, the structure mean RMSE in the absence of noise were $2.5 \%$ and $0.0 \%$ for MSO and MB respectively. With noise the MSO and MB structure errors were $4.7 \%$ and $3.0 \%$ ( $\mathrm{p}<0.001)$.

The simulated factors and factors resolved from the images with noise are plotted in Fig. 4. For the simulation of complete blood clearance, MSO and MB agree with the simulation similarly well. In the simulation of residual blood activity MSO results exhibited systematic deviation from the simulated factors with confidence intervals that do not overlap the simulated lines in the early time frames. MB confidence intervals overlapped the simulated curves.

Short axis slices of the resolved structures from the simulations without noise using both MSO and MB are shown in Fig. 3. In general, the myocardium structures reproducibly agreed with the simulated PV images. However, the MSO derived RV blood structure extended incorrectly into the septal wall and LV blood region for the case with complete blood clearance. Likewise, with residual blood activity the MSO derived LV blood structure extended somewhat into the septal wall and RV region, whereas the MB structures agreed more closely with the simulated PV images. With residual blood activity the myocardium:blood contrast (superimposed in white) in the uptake image (29\%) was markedly improved both with MSO (89\%) and MB (82\%), but MB agreed more closely with the simulated myocardium structure (82\%).

The MB method recovered the model parameters accurately in the absence of noise and with modest errors in the presence of noise as shown in Table I. In the absence of noise, the simulated values were recovered to within three significant figures. In the presence of noise the simulated values were within the range of the mean \pm one standard deviation. The largest bias in parameter estimates was $8.3 \%$ for the $\beta$, and the smallest average being $3.5 \%$ for $t_{p}$. The same analysis could not performed on MSO results because the kinetic model parameters are not resolved as part of the decomposition process.

Estimates of $k_{2}$ parameters, $k_{2}$ ', were determined by fitting the kinetic model (equation 8) to the LV blood and myocardium factors. For the complete blood clearance cases $k_{2}$ ' were $0.065 \pm 0.020$ and $0.048 \pm 0.004$ with MSO and MB respectively, and for residual blood activity the corresponding values were $0.486 \pm 0.100$ and $0.478 \pm 0.032$. In all cases the mean did not significantly differ from the simulated values 0.05 and 0.5 
Canine

Complete Blood Clearance
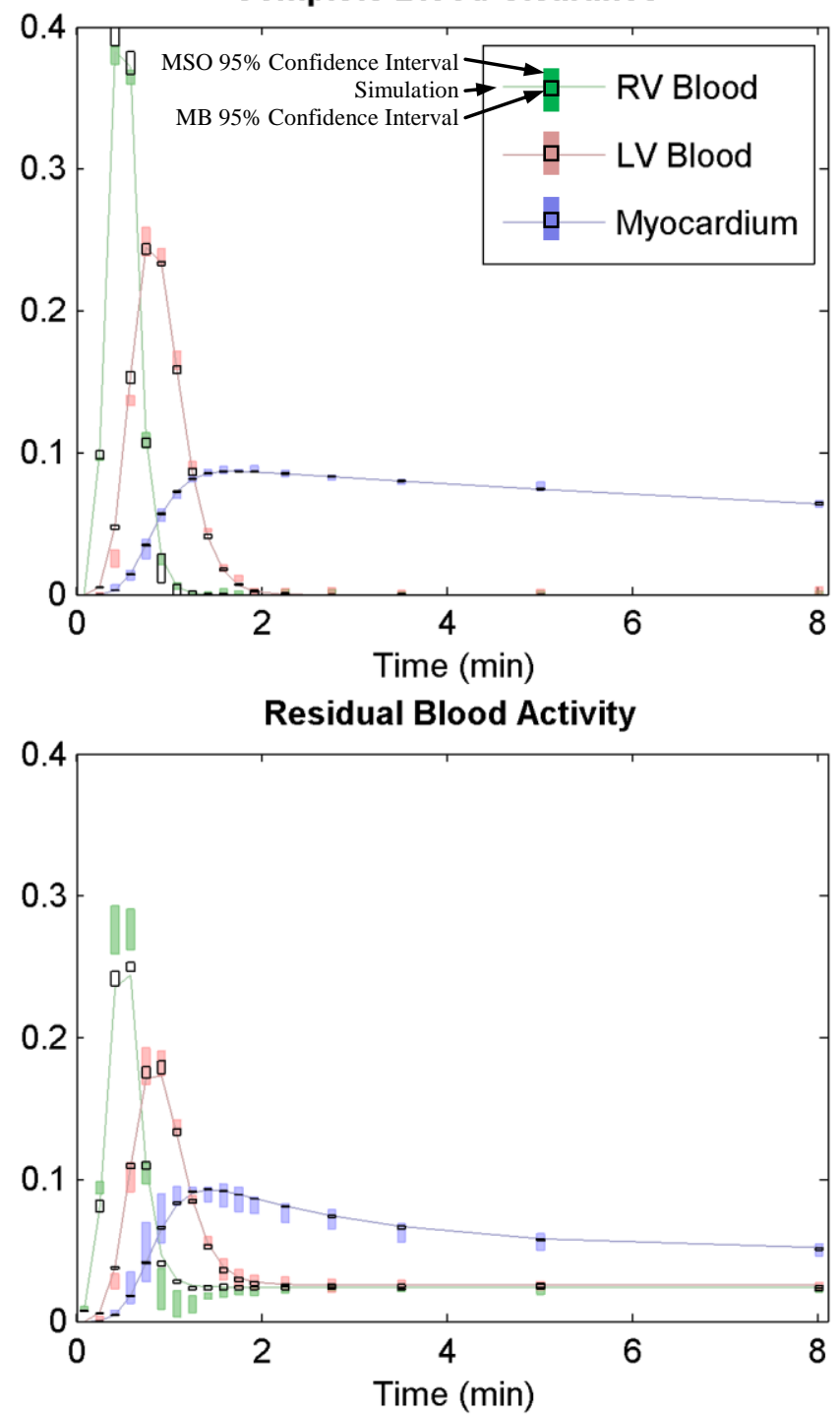

Small Animal

Complete Blood Clearance
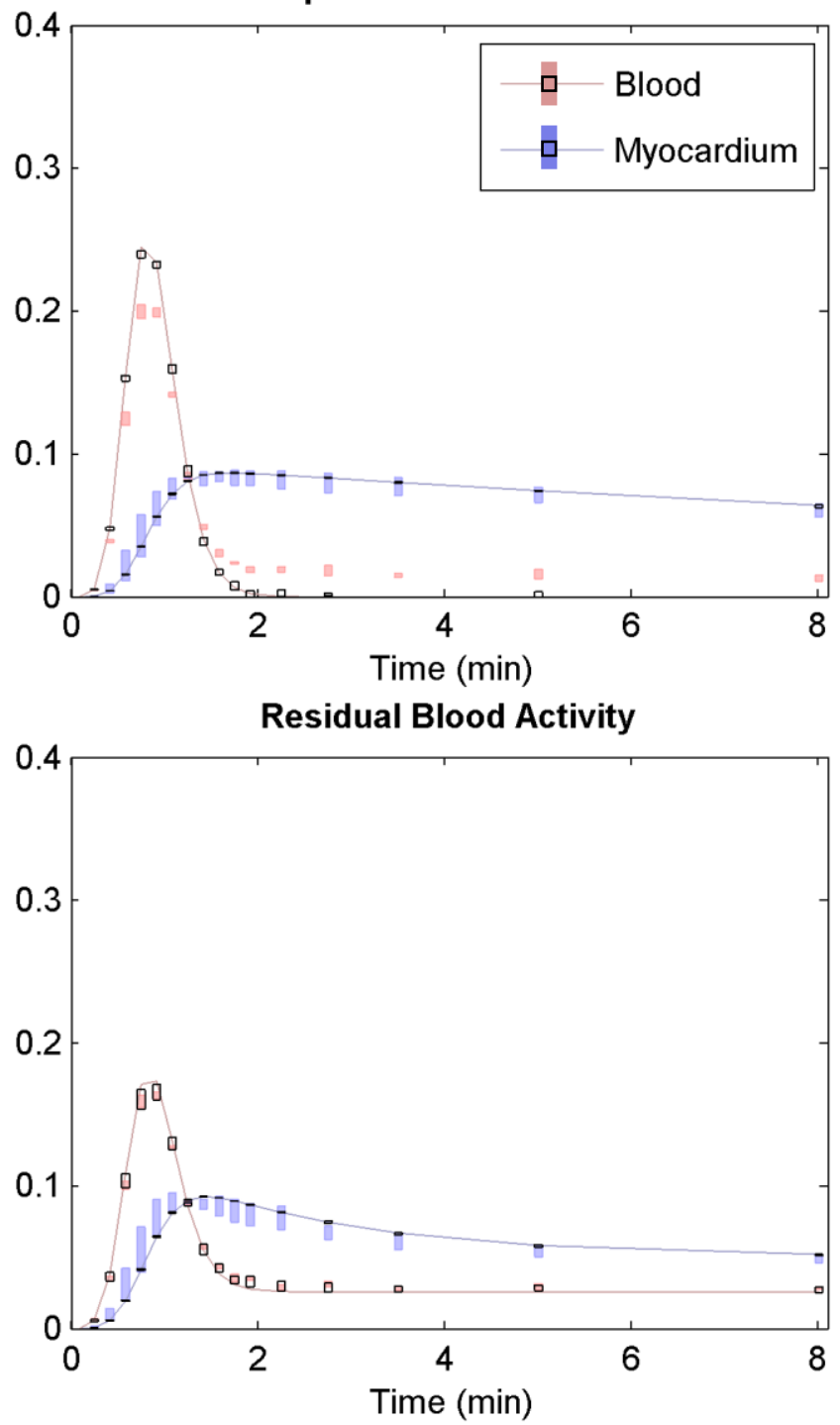

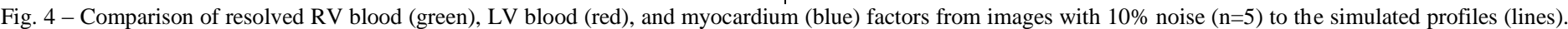

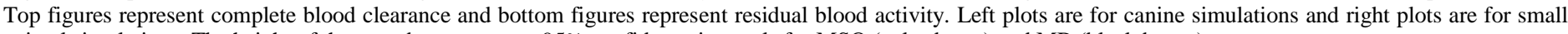
animal simulations. The height of the error bars represent $95 \%$ confidence intervals for MSO (color bares) and MB (black boxes).

(p>0.1). However, the variance of $k_{2}^{\prime}$ with MSO was significantly greater than with $\mathrm{MB}(\mathrm{p}<0.001)$.

Convergence of the MB algorithm was found to be robust regardless of the initial model parameters and is demonstrated by the box plots of $k_{2}$ parameter (Fig. 5) using 5 realizations of noise in the cases of complete blood clearance (C) and residual blood activity (R) for 7 different initial estimate combinations of the model parameters $\left(t_{p}, \beta\right.$, and $\left.k_{2}\right)$. Variances were similar for the complete blood clearance case and identical for the residual blood case. Regardless of the initial estimates, mean $k_{2}$ was not significantly different from the true values (green lines). In addition Fig. 5 shows the $k_{2}$ values from the images without noise as blue stars, all of which deviated less than $0.002 \%$ from the simulated values.

\section{B. Small Animal Simulation}

In the presence of noise, significantly lower RMSE was obtained with MB factors $(\mathrm{p}<0.001)$ and structures $(\mathrm{p}<0.001)$ compared to MSO, as shown in Table III. Both the MB factors

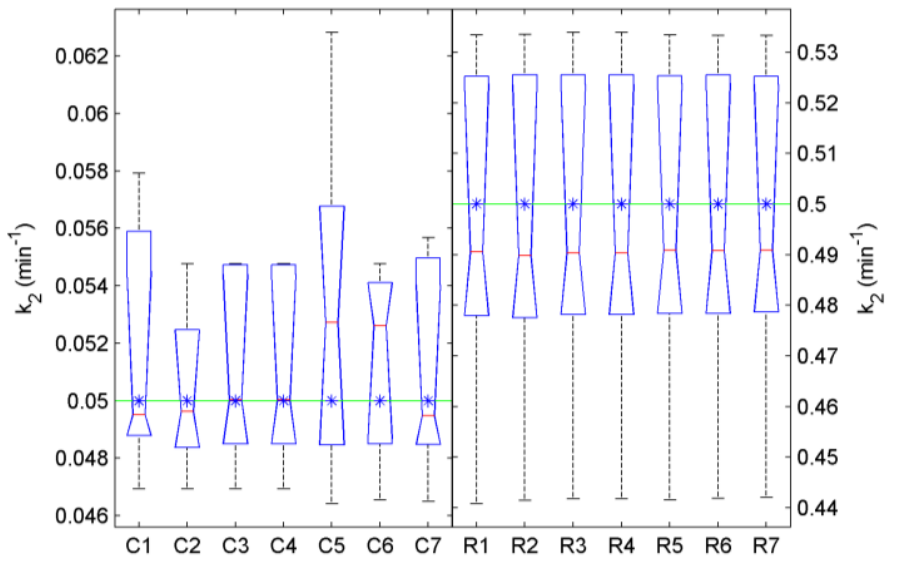

Fig. 5 - Box plots ( $\mathrm{n}=5$ simulated images with noise) of $k_{2}$ parameters with 7 different initial parameter estimates each for complete blood clearance and residual blood activity ( $\mathrm{C}$ and $\mathrm{R}$ respectively). The blue stars are results for $(\mathrm{n}=1)$ case with no noise and are not included in the box plot analysis. 
and structures agreed closely with the simulation data as shown by the estimated structures in Fig. 3 and the factors in Fig. 4.

The MSO derived myocardial structure for the case of complete blood clearance had a noticeable reduction in the septal wall (left part of myocardium) as shown in Fig. 3 and The corresponding blood factors failed to decrease to near zero activity (Fig. 4). In the case of residual blood activity the MSO derived myocardium factors displayed greater activity at early time frames which is typical of contamination by blood signal [6] and activity in the septal wall was also reduced compared to the rest of the myocardium.

The MB derived myocardium factors had myocardium:blood contrast $(46 \%)$ that agreed well with the simulation values (Fig. 3). As expected, MSO increased the contrast to $66 \%$ and $59 \%$ respectively for the cases of complete blood clearance and residual blood activity. Both methods significantly improved contrast over that of the uptake image $(16 \%)$ in the case of residual blood activity.

\section{Experimental Factor Validation with Arterial Sampling}

Comparisons of blood factors obtained using MSO and MB are shown in Fig. 6 for all six rats. Various injection profiles were used: fast bolus (a and f), slow bolus (b), dual injections (c, $\mathrm{d}$, and e). Both MB and MSO showed reasonable correspondence with the sampled blood curves in the first 2-3 minutes, but systematically differed in the late time frames (uptake phase). The MSO derived blood factor consistently had residual blood activity, while MB derived blood factors consistently dropped to near zero activity. Blood sampled curves consistently dropped to near zero activity, but not as fast as the MB blood factors did.

Mean RMSE values were $2.2 \pm 0.7 \%$ and $1.6 \pm 0.4 \%$ with MSO and $\mathrm{MB}$ respectively (shown in the box plots of Fig. 7). The mean RMSE with MB was significantly lower than with MSO $(\mathrm{p}=0.027)$, but the variance was not $(\mathrm{p}=0.3)$.

\section{Structure Validation with ${ }^{11}$ CO Blood Pool Imaging}

The number of resolved factors was consistently 2 for long tracer infusions $(\geq 120 \mathrm{~s})$ and 3 for short infusions $(\leq 30 \mathrm{~s})$. Where

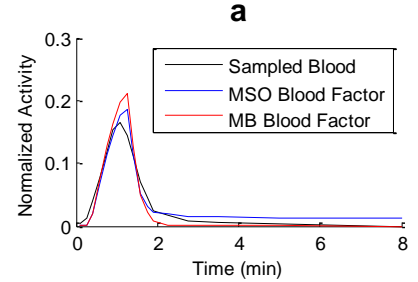

C

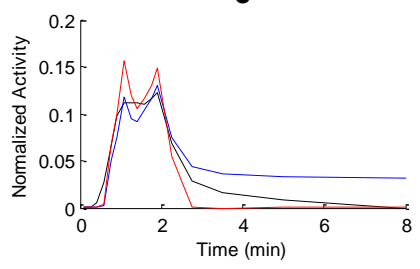

e
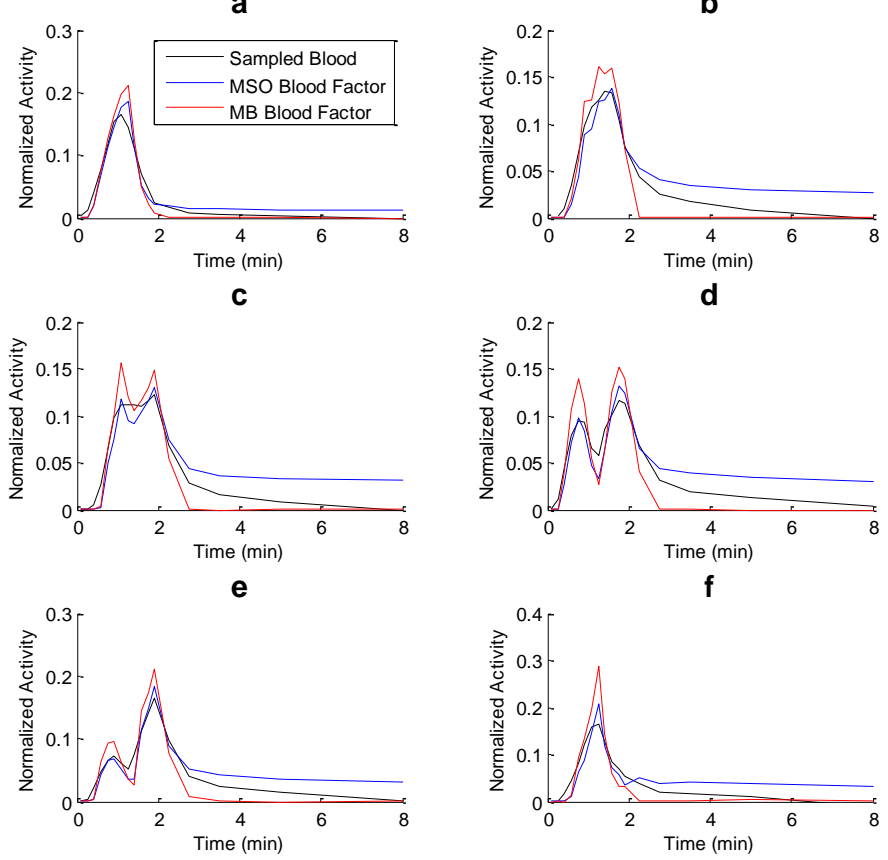

d

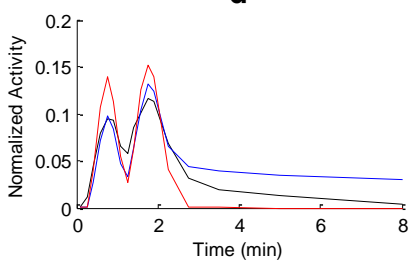

f

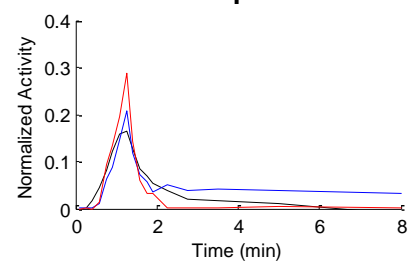

Fig. 6 - Blood factors obtained using both MSO (blue) and MB (red) methods compared to blood sampled curve (black). All curves are normalized to unity area.

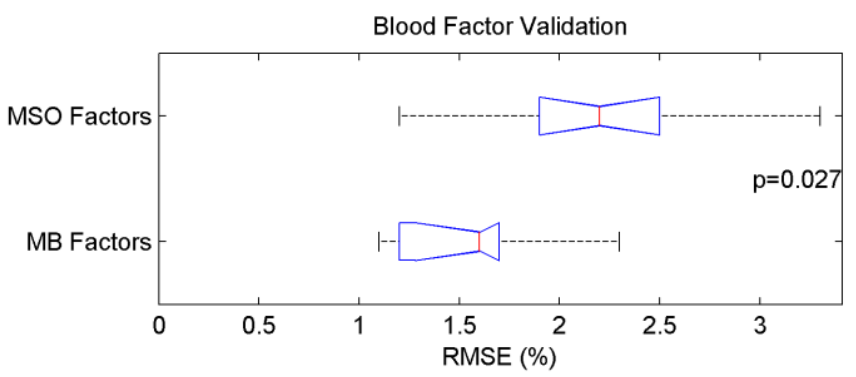

Fig. 7 - Comparison of MSO and MB derived blood factors to arterial blood sampled curves.

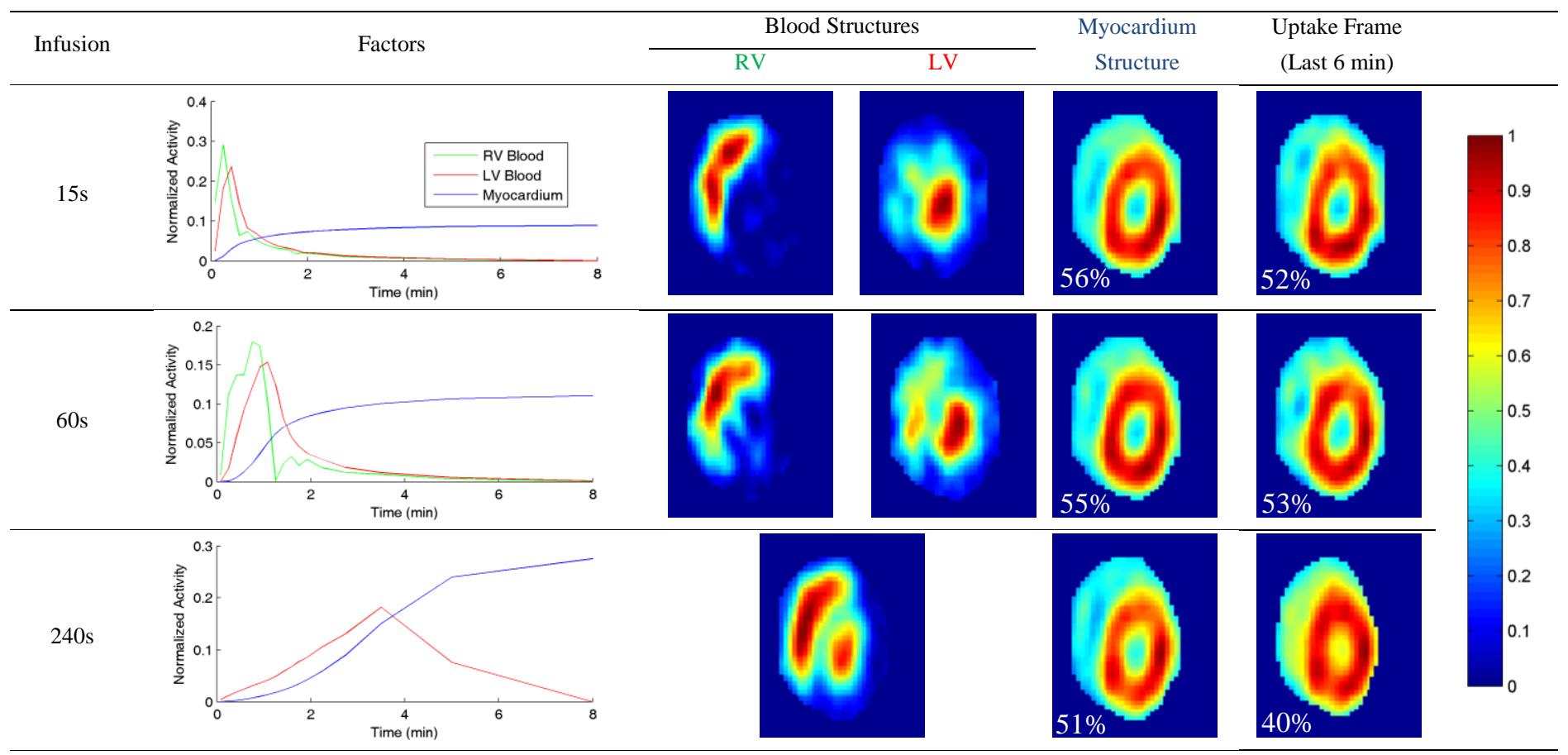

Fig. 8 - Example short axis slices of resolved factors and structures, and uptake (last 6 min) frames for a 15s, 60s, and 240s infusion duration example in a dog. 
3 factors were resolved, spatially distinct RV and LV structures were observed as demonstrated in Fig. 8.

Factors were representative of the infusion duration. Blood factors had a more gradual rise and delayed clearance with prolonged infusions. Likewise myocardial uptake was more gradual as expected. In all cases, blood activity cleared almost completely at the last time point, as demonstrated by the factors in Fig. 8.

Agreement of the blood structures with the ${ }^{11} \mathrm{CO}$ blood pool image of the same animal was good, with RMSE $<9.7 \%$ in all cases for both MSO and MB. Box plots of the RMSE are shown in Fig. 9, indicating no significant differences in RMSE ( $p=0.23$ ) with $\mathrm{MB}$ compared to MSO values. No obvious trends in RMSE with infusion duration were observed with either method (data not shown). No significant difference was detected in the variability of RMSE $(\mathrm{p}=0.8)$ between MSO and MB.

\section{E. Structure Reproducibility using Variable Tracer Infusion Durations}

Reproducibility of structures was good to excellent. For all RMSE combinations $(n=45)$, RMSE was $\leq 11.0 \%$ with MSO and $\leq 7.2 \%$ with MB. Box plots of the RMSE values for blood and myocardium structure reproducibility are shown in Fig. 10. Mean myocardial RMSE was $6.2 \%$ with MSO vs. $3.9 \%$ with $\mathrm{MB}(\mathrm{p}<0.01)$, and in blood structures respective mean RMSE values were $5.6 \%$ vs $4.9 \% \quad(p=0.006)$. The single outlier (red cross) was in MSO blood RMSE between a 15 and a $60 \mathrm{sec}$ infusion. Visual comparison of the structures in this study with others did not reveal an obvious difference in spatial patterns and a single outlier from 45 sets can be expected due to random distribution.

Myocardium:blood contrasts were 49 $\pm 5 \%, 62 \pm 7 \%$, and $52 \pm 3 \%$ in the uptake images, MSO myocardial structures, and MB myocardial structures respectively as demonstrated by the corresponding contrast superimposed on the images in Fig. 8. Mean contrasts using either MSO or MB were significantly higher than in the uptake images $(\mathrm{p}<0.001$ for MSO and $\mathrm{p}=0.04$ for $\mathrm{MB})$. The variance in the contrast across the 10 images tended to be reduced with MB compared to MSO $(\mathrm{p}=0.062)$ indicating better reproducibility.

The mean CV\% ( $n=75,782$ pixels) across the 10 myocardial structures was $12.9 \%$ and $6.5 \%$ for MSO and MB respectively. Across the 10 blood factors respective CV\% was $16.8 \%$ and $11.4 \%$. The combined CV\% for myocardium and blood structures was $12.9 \%$ for MSO and $8.9 \%$ for MB. All the differences between MSO and MB were significant $(\mathrm{p}<0.001)$ indicating the $\mathrm{MB}$ resolved more reproducible structures in the same animal with varying tracer infusion.

\section{F. Global MBF Reproducibility using Variable Tracer Infusion Durations}

MBF quantification and estimates (shown in Table IV) with MB tended towards smaller variability than with MSO, as

TABLE IV

Reproducibility of MBF Quantification - Mean \pm standard-deviation (CV\%)

\begin{tabular}{|c|r|r|r|r|r|}
\hline \multirow{2}{*}{} & \multicolumn{2}{c|}{ ROI } & \multicolumn{2}{c|}{ Without RV Blood } & \multicolumn{2}{c|}{ With RV Blood } \\
\cline { 3 - 6 } \multicolumn{2}{|c|}{} & \multicolumn{1}{c|}{ MSO } & \multicolumn{1}{c|}{ MB } & \multicolumn{1}{c|}{ MSO } & \multicolumn{1}{c|}{ MB } \\
\hline \multirow{2}{*}{ K1 } & $0.58 \pm 0.06$ & $0.76 \pm 0.08$ & $0.78 \pm 0.05$ & $0.77 \pm 0.08$ & $0.80 \pm 0.05$ \\
& $(10 \%)$ & $(11 \%)$ & $(7 \%)$ & $(10 \%)$ & $(6 \%)$ \\
\hline \multirow{2}{*}{ MBF } & $0.98 \pm 0.17$ & $1.56 \pm 0.30$ & $1.65 \pm 0.21$ & $1.61 \pm 0.29$ & $1.71 \pm 0.18$ \\
& $(17 \%)$ & $(19 \%)$ & $(13 \%)$ & $(18 \%)$ & $(10 \%)$ \\
\hline
\end{tabular}

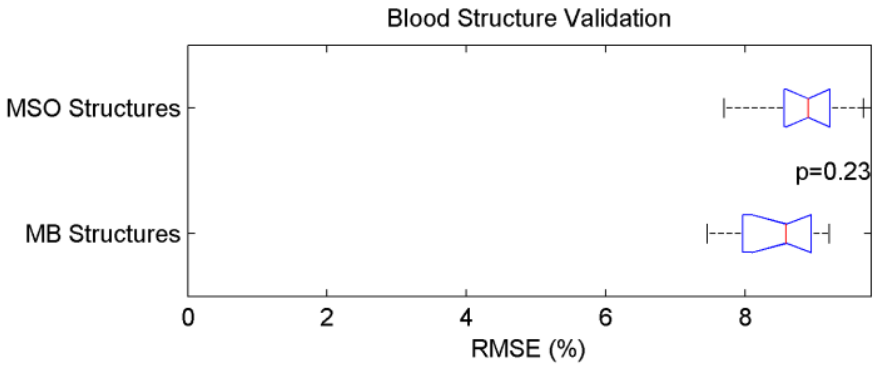

Fig. 9 - Comparison of MSO and MB derived blood structures to ${ }^{11} \mathrm{CO}$ blood-pool images $(\mathrm{CO})$.

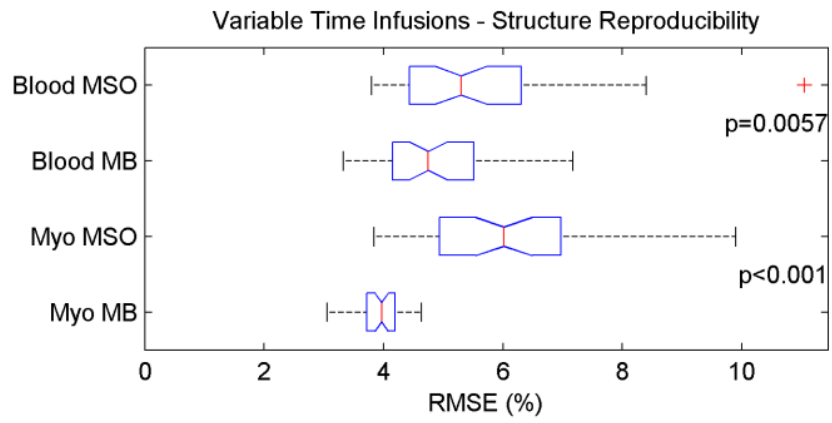

Fig. 10 - Reproducibility of blood structures (top two rows) and myocardium structures (bottom two rows) obtained from variable duration infusions using MSO and MB.

indicated by small CV\% values, but did not reach statistical significance $(p=0.2$ and $p=0.15$ without and with $R V$ respectively for $\mathrm{K} 1$, and $\mathrm{p}=0.32$ and $\mathrm{p}=0.16$ for $\mathrm{MBF}$ ). Amplification of the MBF variability compared to that of $K_{l}$ is expected due to the non-linear relationship of the extraction function. Both with MSO and MB factors, variability in global $K_{l}$ and $\mathrm{MBF}$ estimates did not improve significantly $(\mathrm{p}>0.7)$ with the addition of the RV blood in the model.

Mean $K_{l}$ and MBF values both with MSO and MB factors were significantly higher than ROI based values ( $p$-value $<0.001)$ indicating that the extraction function may not be applicable for quantification using factors.

\section{DISCUSSION}

We developed, validated, and compared two dynamic image decomposition methods, MSO and MB. These methods were implemented in an identical manner with the exception of the constraints imposed on the solution so as to resolve the nonuniqueness problem. The previously reported MSO method [14][16][17] constrained the solution by minimizing the spatial overlap between structures. The method described in this work, $\mathrm{MB}$, constrained the solution by use of kinetic models between the factors, and penalizing residual blood activity and rise in tracer blood concentrations in the uptake phase of the image to ensure uniqueness of the solution.

Simulation results clearly showed that the MB method outperforms MSO for accuracy in recovering factors and structures, particularly in small hearts where the limited resolution of PET leads to greater spatial overlap between structures. The small animal simulations demonstrated that MSO's inability to separate myocardium and blood signals can result in less accurate blood factors and myocardium factors contaminated by blood signal. In addition MSO derived myocardium structures may suffer from reduced intensity in the septal wall which could be misinterpreted as a defect. However 
the higher myocardium to blood pool contrast of MSO derived myocardium structures may be beneficial for registration and segmentation purposes. If regions of pure blood exist in the image, such as in large hearts, MSO and MB are both expected to resolve accurate blood factors that have less noise than ROI based methods. Scatter in the image may be regarded as an additional, broad blurring kernel, in which case we expect MB to give more accurate blood factors than MSO.

The experimental results largely agreed with simulations and suggested that MB can be more accurate and more reproducible than MSO. Blood factors were more accurate when derived with $\mathrm{MB}$ as indicated by arterial blood sampling. However, comparison of blood structures to blood pool imaging did not demonstrate superiority of either MSO or MB.

\section{A. Residual Signal}

The premise of dynamic image decomposition is that most of signals in the image are represented by the factors and that the residual signal contains a combination of noise and smaller signals that can be disregarded. Fig. 11 demonstrates the residue in decomposition of the canine simulation with no noise and complete blood clearance using MSO and MB. The three columns (left to right) show the original image sequence, the scaled structures that compose the image, and the residue. Both with MSO and MB the residue is random and is 5 orders of magnitude smaller than the dynamic image sequence. This qualitative information indicates good convergence using both methods as the combined factors and structures accurately represent the dynamic sequences in the image. This supports the notion that any difference in results between $\mathrm{MSO}$ and $\mathrm{MB}$ are a result of the constraints rather than implementation of the optimization routines.

\section{B. Penalty Weights}

Both MSO and MB cost functions $\left(C_{M S O}\right.$ and $C_{M B}$ in equation (2) and (10) respectively) consist of weighted penalties. The respective weights were adjusted empirically using the simulated data to ensure convergence and good correspondence of factors and structures with the simulated data. Since the respective penalties are all bound between 0 and 1, their weights somewhat determine their relative importance. We prioritized the MSO penalties in order of non-negative factors, non-negative structures, and minimal spatial overlap. For MB, the penalties
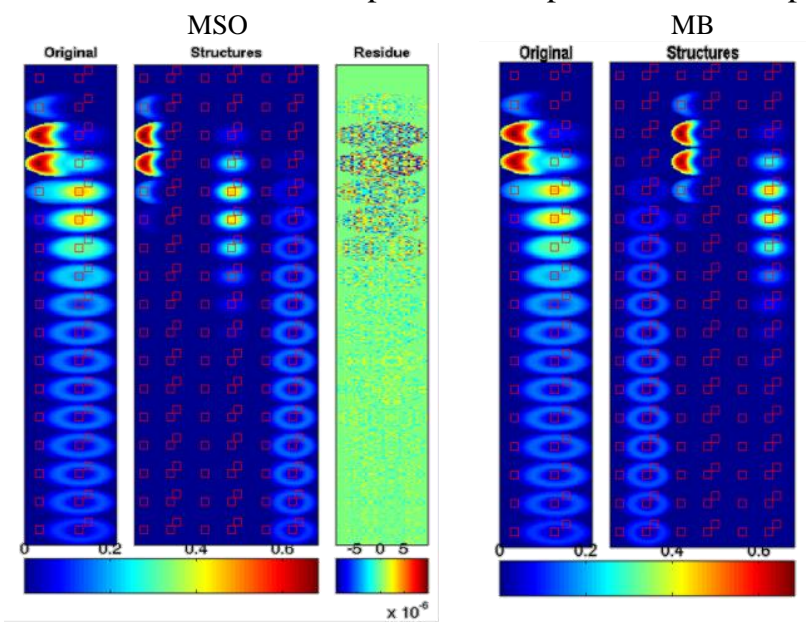

Fig. 11 - Decomposition results showing original image sequence as a series of frames from top to bottom, the scaled structures for all time frames, and the residual signal for MSO (left) and MB (right) for the noise-free total blood clearance case. The residue image using both methods is completely random and on the scale of $10^{-6}$. were prioritized in order of non-negative factors, no rise in blood activity during the uptake phase, non-negative structures, and minimal residual blood activity in the uptake phase. In order to test the sensitivity the chosen parameters, we varied the weights over 1 order of magnitude and found that the results changed little (data not shown). A limitation of this work is that automatic parameter selection was not demonstrated. Future work should evaluate the current penalty weights and/or tune them.

\section{Execution Time}

In 30 of the 40 images used in this work, the execution time with MB was shorter than with MSO. On average the execution times ratio (MSO/MB) was 2.4 \pm 2.4 . Although $\mathrm{MSO}$ requires less computation per iteration compared to $\mathrm{MB}$, MSO has more free parameters to optimize. The median execution time with MSO was 9.6 hours and 5.8 hours with MB on a modern PC. In this work, there was little emphasis on performance and more on precision, however there are several potential possibilities for accelerating execution, such as using a subset of pixels and reducing the tolerances on the optimization stopping criteria. A more efficient implementation in a non-interpreter based environment such as $\mathrm{C}$ language could also shorten execution time substantially.

\section{Blood Clearance}

Previous studies demonstrated that blood clearance results can vary with different decomposition methods and constraints [18]. The purpose of simulating data with and without blood clearance was to ensure that no bias existed in our solution. The results of Fig. 4 demonstrate an ability to reliably resolve both scenarios using the MB method even in the presence of noise. The blood factors obtained from real images tended towards complete blood clearance which agrees with previous observations [29].

The MSO method did not reliably result in clearance of activity form the blood factors. In the canine simulation where the structures were relatively large compared to feature sizes blood clearance was resolved correctly. However, this was not the case in the small-animal simulation where the relatively larger blur resulted in no pixels with pure blood signal. In the rat experiments, residual blood activity was observed in all images, agreeing with the simulation results. One could expect more accurate results with MSO in other organs without substantial blood contamination [14]. However in small animal studies and humans with small hearts MSO may be biased, especially with the high positron range of ${ }^{82} \mathrm{Rb}$.

\section{E. ${ }^{11}$ CO Blood Pool Images}

Although MB derived blood structures tended to agree better with ${ }^{11} \mathrm{CO}$ blood pool images than MSO derived blood structures, no significant difference was found $(p=0.23)$. Qualitatively, MSO blood structures were more defined, having a smaller LV and RV cavity regions and a larger gap in the septal wall region as demonstrated for a $50 \mathrm{sec}$ elution results in Fig. 8. The MSO myocardium factors (blue line in Fig. 12) had greater activity in the early time frames than the MB derived factor (dashed blue line), consistent with blood signal contamination [6]. Thus MSO reduces spatial overlap of myocardium and blood structures by attributing the blood signal in the myocardium region to myocardial signal.

With either decomposition method, correspondence between blood structures and ${ }^{11} \mathrm{CO}$ blood pool images (Fig. 9) was worse than between pairs of blood structures (Fig. 10) as reflected by 
significantly greater RMSE values $(\mathrm{p}<0.001)$. The ${ }^{11} \mathrm{CO}$ image, which was taken on a separate occasion, was translated spatially to align with the blood factors, but no rotation correction was applied. In addition, ${ }^{82} \mathrm{Rb}$ images may suffer from bias due to $777 \mathrm{keV}$ prompt gammas that may not be fully corrected [30]. Image spatial resolution may also be slightly different due to positron range even though complimentary smoothing was applied to the ${ }^{11} \mathrm{CO}$ image.

While comparison of structures under different infusion durations enables good reproducibility measurement it does not ensure physiological accuracy. It is desirable to improve the correspondence with $\mathrm{CO}$ images. Repeating the experiment with $\mathrm{CO}$ imaging on the same session as ${ }^{82} \mathrm{Rb}$ may be beneficial for optimal evaluation. Also labeling $\mathrm{CO}$ with ${ }^{15} \mathrm{O}$ instead of ${ }^{11} \mathrm{C}$ could more closely reproduce ${ }^{82} \mathrm{Rb}$ resolution loss due to the longer positron range.

\section{F. ${ }^{82} \mathrm{Rb}$ Blood Sampling in Rats}

The use of ${ }^{82} \mathrm{Rb}$ as an imaging agent in rats is not ideal due to the combination of small anatomy and large positron range. As a result the image resolution is on the same order (or less) as the size of the imaged organ. Rats were chosen to achieve sufficiently high tracer concentrations in the blood for the microvolumetric sampler to have adequate precision. This was not possible in larger animals since their larger blood volume significantly dilutes tracer concentrations. More sensitive blood sampling equipment would enable similar experiments in larger animals in which imaging conditions are more favorable.

Although the heart walls could not be resolved in the reconstructed images, it is impressive to note that reasonable decomposition could still be achieved using both MB and MSO methods as demonstrated by the results of Fig. 6 and Fig. 7.

MSO blood factors always demonstrated residual blood activity in the late time frames, which was consistent with inaccuracies in the small animal simulations. MB blood factors on the other hand showed complete blood clearance which agreed more closely with the blood samples. Nevertheless, a sudden drop to near zero activity in the MB blood factors is apparent in the rat data, which may indicate that the residual blood penalty is too severe. As mentioned above, penalty weights were manually adjusted and MB could potentially benefit of a more rigorous tuning including the penalty weight and the number of frames which constitute the uptake phase.

\section{G. ${ }^{82}$ Rb Infusion Duration}

Optimal ${ }^{82} \mathrm{Rb}$ infusion duration for perfusion quantification has been explored previously [31]. While mean perfusion values did not change with infusion durations, regional variability decreased with longer infusions (possibly increasing quantification accuracy). The disadvantage of long infusions, however, is that the uptake image, reported clinically, suffers from reduced myocardium to blood contrast (Fig. 8). The reduction in contrast is due to insufficient time for complete tracer clearance from the blood. The myocardium structures obtained with model-based factor analysis can recover image contrast by removing blood signal contribution. Model-based factor analysis may make long ${ }^{82} \mathrm{Rb}$ infusions practical, with improved perfusion quantification and high contrast myocardial structure images for routine clinical applications.

\section{H. $M B$ with Other Tracers}

To the best of our knowledge, all previous PET image decomposition methods were not tracer specific. While the onecompartment model used in this work is also applicable to other tracers, such as ammonia [32], acetate [33], and HED [34], the kinetics of some tracers may be better modeled with a twocompartment model. The MB method is compatible with twocompartment kinetic models as well as blood metabolite corrections, however further validation is required.

\section{Number of Factors}

While MSO can be used to resolve any number of components, $\mathrm{MB}$ assumes the existence of specific types of components in the image. In this work we dealt with solving one blood component and one myocardium component as well as two blood components and one myocardium component. Additional tissue components (such as stomach or liver) could be accommodated with additional factors with a response function as in equation 8 or a different model and associated model parameter constraints. Models need to be customized for the tracer and organ in question. Regardless of implementation, the temporal response of additional tissues would have to be sufficiently different from that of the myocardium to obtain reliable component separation.

\section{J. Application of FA to Quantification of Myocardial \\ Physiology}

Regardless of the decomposition method, it is assumed that factors represent uniform temporal responses of image components. This has raised concerns, particularly in diseased myocardium, where the temporal responses of diseased and healthy regions may vary. In MB this issue is manifested by resolving a single washout parameter, $k_{2}$, which is assumed constant for the entire myocardium. To resolve this issue we and others [16][17] propose to use the blood factors as the input functions to the kinetic model, and ROI sampled myocardial TACs as the output functions. The disadvantage of using ROI derived output function compared to myocardium factors is that they contain noise

Quantification of MBF using ${ }^{82} \mathrm{Rb}$ and kinetic modeling requires the implementation of an extraction fraction correction [35][36] which is calibrated to a standard [19]. In the variable infusion duration results it was evident that the extraction correction function was calibrated for the ROI based method, but

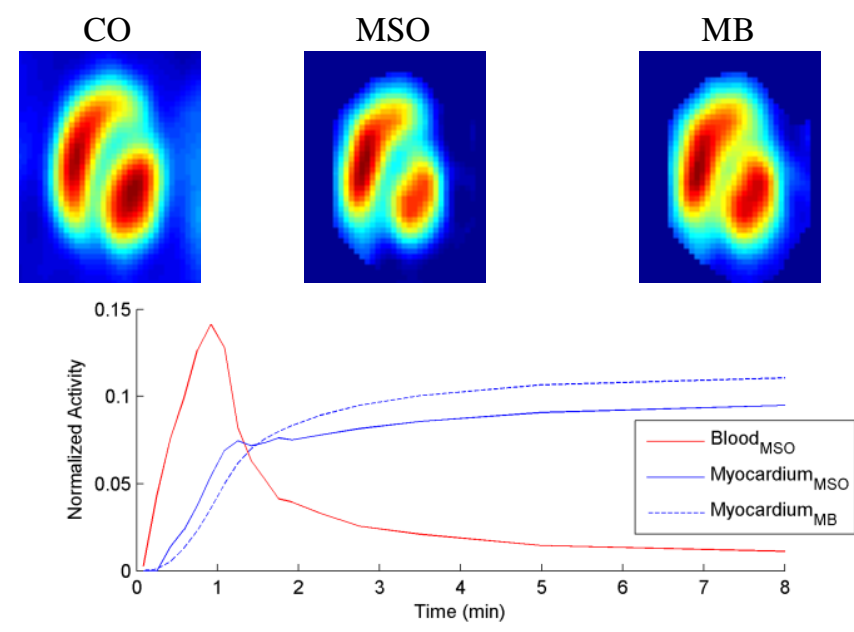

Fig. 12 - Example short axis slice through blood pool images using CO imaging, and through MSO and MB derived structures from the same $60 \mathrm{sec}$ infusion image shown in Fig. 8. Factors for MSO are shown below, with the myocardium factor from Fig. 8 (dashed line). 
not when factors were used as the input function (MBF changed from $\sim 0.98$ with ROI to $\sim 1.65$ with factors). Calibration was not possible in this case since we did not have data that spans the range of physiologic flow values. Thus future work on MBF quantification with factors analysis should include calibration of the extraction function to a standard.

\section{CONCLUSION}

Constraints must be placed on dynamic cardiac PET image decomposition in order to resolve physiologically accurate factors. We used simulation and experimental data to compare the physiological accuracy of the MSO and MB methods. Factors and structures obtained using $\mathrm{MB}$ agreed with simulations significantly better than MSO. In experimental ${ }^{82} \mathrm{Rb}$ PET data MB agree better than MSO with the physiological standards of arterial blood sampling. In addition, MB had more reproducible structure images and tended towards more reproducible global myocardial blood flow measurements with varying tracer infusion durations. The potential benefit of model-based factor analysis for quantification of myocardial blood flow should be explored in future validation studies.

\section{REFERENCES}

[1] K. Yoshinaga, B. J. W. Chow, K. Williams, L. Chen, R. A. deKemp, L. Garrard, A. L.-T. Szeto, M. Aung, R. A. Davies, T. D. Ruddy, and R. S. B. Beanlands, "What is the prognostic value of myocardial perfusion imaging using rubidium-82 positron emission tomography?," J. Am. Coll. of Cardiol., vol. 48, no. 5, pp. 1029-1039, 2006.

[2] P. A. Kaufmann and P. G. Camici, "Myocardial blood flow measurements by PET: Technical aspects and clinical applications," J. Nuc. Med., vol. 46, no. 1, pp. 75-88, 2005.

[3] R. A. Tio, A. Dabeshlim, H.-M. J. Siebelink, J. de Sutter, H. L. Hillege, C. J. Zeebregts, R. A. J. O. Dierckx, D. J. van Veldhuisen, F. Zijlstra, and R. H. J. A. Slart, "Comparison between the prognostic value of left ventricular function and myocardial perfusion reserve in patients with ischemic heart disease," J. Nucl. Med., vol. 50, no. 2, pp. 214-9, 2009.

[4] K. Zierler, "A critique of compartmental analysis," Ann. Rev. Biophys. Bioeng., vol. 10, pp. 531-562, 1981.

[5] R. S. Beanlands, S. Thorn, J. N. DaSilva, T. Ruddy, and J. Maddahi, "Myocardial Viability," in Principles and Practice of Positron Emission Tomography, second edition, R. Wahl, Ed. Philadelphia: Lippincott Williams \& Wilkins, 2009.

[6] J. Kim, P. Herrero, T. Sharp, R. Laforest, D. J. Rowland, Y.C. Tai, J. S. Lewis, and M. J. Welch, "Minimally invasive method of determining blood input function from PET images in rodents," J. Nucl. Med., vol. 47, no. 2, pp. 330336, 2006.

[7] F. Hermansen and A. A. Lammertsma, "Linear dimension reduction of sequences of medical images: I. Optimal inner products," Phys. Med. Biol., vol. 40, pp. 1909-1920, 1995.
[8] H.-M. Wu, C. K. Hoh, Y. Choi, H. R. Schelbert, R. A. Hawkins, M. E. Phelps, and S.-C. Huang, "Factor analysis for extraction of blood time-activity curves in dynamic FDG-PET studies," J. Nucl. Med., vol. 36, no. 9, pp. 17141722, 1995.

[9] C. Schiepers, C. K. Hoh, J. Nuyts, H.-M. Wu, M. E. Phelps, and M. Dahlbom, "Factor Analysis in Prostate Cancer: Delineation of Organ Structures and Automatic Generation of In- and Output Functions," IEEE Trans. Nucl. Sci., vol. 49, no. 5, pp. 2338-4233, 2002.

[10] H. Benali, I. Buvat, F. Frouin, J. P. Bazin, and R. Di Paolo, "Foundations of factor analysis of medical image sequences: a unified appraoach and some practical implications," Img. and Vis. Comp., vol. 12, no. 6, pp. 375-385, 1994.

[11] A. Hyvärinen and E. Oja, "Independent Component Analysis Algorithms and Applications," Neural Networks, vol. 13, no. 4-5, pp. 411-430, 2000.

[12] I. Buvat, H. Benali, F. Frouin, J. P. Banzin, and R. Di Paola, "Target apex-seeking in factor analysis of medical image sequences," Phys. Med. Biolo., vol. 38, pp. 123-138, 1993.

[13] I. Buvat, H. Benali, and R. Di Paola, "Statistical Distribution of Factors and Factor Images in factor Analysis of Medical Image Sequences," Phys. Med. Biol., vol. 43, pp. 1695-1711, 1998.

[14] A. Sitek, G. T. Gullberg, and R. H. Huesman, "Correction for Ambiguous Solutions in Factor Analysis Using Penalized Least Squares Objectives," IEEE Trans. Med. Imaging, vol. 21, no. 3, pp. 216-225, 2002.

[15] A. Sitek, E. V. R. Di Bella, G. T. Gullberg, and R. H. Huesman, "Removal of Liver Activity Contamination in Teboroxime Dynamic Cardiac SPECT Imaging with the Use of Factor Analysis," J. Nucl. Cardiol., vol. 9, no. 2, pp. 197205, 2002.

[16] G. El Fahkri, A. Sitek, B. Guérin, M. F. Kijewski, M. F. Di Carli, and S. C. Moore, "Quantitative Dynamic Cardiac 82Rb PET Using Generalized Factor and Compartment Analyses," J. Nuc. Med, vol. 46, no. 8, pp. 1264-1271, 2005.

[17] G. El Fakhri, A. Kardan, A. Sitek, S. Dorbala, N. AbiHatem, Y. Lahoud, A. Fischman, M. Coughlan, T. Yasuda, and M. F. Di Carli, "Reproducibility and accuracy of quantitative myocardial blood flow assesment with $82 \mathrm{Rb}$ PET: Comaprison with 13N-ammonia PET," J. Nucl. Med., vol. 50, no. 7, pp. 1062-1071, 2009.

[18] R. Klein, M. Bentourkia, R. R. S. Beanlands, A. Adler, and R. A. deKemp, "A minimal factor overlap method for resolving ambiguity in factor analysis of dynamic cardiac PET," in Nucl. Sci. Symp. Conf. Record, vol. 5, Honolulu, HI, 2007, pp. 3268-3272.

[19] M. Lortie, R. S. B. Beanlands, K. Yoshinaga, R. Klein, J. N. DaSilva, and R. A. deKemp, "Quantification of Myocardial Blood Flow with 82Rb Dynamic PET Imaging," Eur. J. Nucl. Med. Mol. Imaging, vol. 34, no. 11, pp. 1765-1774, 2007.

[20] R. Klein, R. S. Beanlands, A. Adler, and R. A. deKemp, "Model-based factor analysis of dynamic sequences of cardiac positron emission tomography," in Nucl. Sci. Symp. Conf. Record, Dresden, Germany, October 2008, pp. 51985202. 
[21] M. T. Madsen, "A Simplified Formulation of the Gamma Variate Function," Phys. Med. Biol., vol. 37, no. 7, pp. 1597-1600, 1992.

[22] M. D. Harper and M. L. Lecklitner, "Derivation of gamma variate indicator dilution function from simple convective dispersion model of blood flow," Med. Phys., vol. 11, no. 5, pp. 690-692, 1984.

[23] R. Davenport, "The Derivation of the Gamma-Variate Relationship for Tracer Dilution Curves," J. Nucl. Med., vol. 24, no. 10, pp. 945-948, 1983.

[24] H. K. Thompson, C. F. Starmer, R. E. Whalen, and H. D. McIntosh, "Indicator Transit Time Considered as a Gamma Variate," Circ. Res., vol. 14, no. 6, pp. 502-515, 1964.

[25] Q. Jinyi and R. M. Leahy, "Resolution and noise properties of MAP reconstruction for fully 3-D PET," IEEE Trans. Med. Imaging, vol. 19, no. 5, pp. 493-506, 2000.

[26] L. Convert, G. Morin-Bassard, J. Cadorette, M. Archambault, M. Bentourkia, and R. Lecomte, "A New Tool for Molecular Imaging: The Microvolumetric $B$ Blood Counter," J. Nucl. Med., vol. 48, no. 7, pp. 1197-1206, 2007.

[27] R. Klein, A. Adler, R. S. Beanlands, and R. A. deKemp, "Precision-controlled elution of a $82 \mathrm{Sr} / 82 \mathrm{Rb}$ generator for cardiac perfusion imaging with positron emission tomography," Phys. Med. Biol., vol. 52, no. 3, pp. 659-673, 2007.

[28] R. Klein, M. Lortie, A. Adler, R. S. Beanlands, and R. A. deKemp, "Fully automated software for polar-map registration and sampling from PET images," Nucl. Sci Symp. and Med. Imag. Conf. Record, pp. 3185-3188, 2006.

[29] I. N. Weinberg, S. C. Huang, E. J. Hoffman, L. Araujo, C. Nienaber, M. Grover-McKay, M. Dahlbom, and H. Schelbert, "Validation of PET-acquired input functions for cardiac studies," J. Nucl. Med., vol. 29, no. 2, pp. 241-247, 1988.

[30] C. Watson, C. Hayden, M. Casey, J. Hamill, and B. Bendriem, "Prompt gamma correction for improved quantification in 82Rb PET," J. Nucl. Med., vol. 49, p. 64P, 2008.

[31] R. deKemp, R. Klein, M. Lortie, and R. Beanlands, "Constant-Activity-Rate Infusions for Myocardial Blood Flow Quantification with 82Rb and 3D PET," Nucl. Sci. Symp. Conf. Record, vol. 6, pp. 3519-3521, 2006.

[32] T. R. DeGrado, M. W. Hanson, T. G. Turkington, D. M. Delong, D. A. Brezinski, J.-P. Vallée, L. W. Hedlund, J. Zhang, F. Cobb, M. J. Sullivan, and R. E. Coleman, "Estimation of myocardial blood flow for longitudinal studies with $13 \mathrm{~N}$-labeled ammonia and positron emission tomography," J. Nucl. Med., vol. 3, no. 6, pp. 494-507, 1996.

[33] J. ven den Hoff, W. Burchert, H. G. Wolpers, G. J. Meyer, and H. Hundeshagen, "A kinetic model for cardiac PET with [1-carbon-11]-acetate," J. Nucl. Med., vol. 37, no. 3, pp. 521-529, 1996.
[34] T. Wichter, M. Schäfers, C. G. Rhodes, M. Borggrefe, H. Lerch, A. A. Lammertsma, F. Hermansen, O. Schober, G. Breithardt, and P. G. Camici, "Abnormalities of cardiac sympathetic innervation in arrhythmogenic right ventricular cardiomyopathy : Quantitative assessment of presynaptic norepinephrine reuptake and postsynaptic $B$-adrenergic receptor density with positron emission tomography," Circ., vol. 101, pp. 1552-1558, 2000.

[35] E. M. Renkin, "Transport of potassium-42 from blood to tissue isolated mammalian skeltal muscles," Am. J. Physiol., no. 197, pp. 1205-1210, 1959.

[36] C. Crone, "Permeability of capillaries in various organs as determined by use of the idicator diffusion method," Acta. Physiol, Scand., no. 58, pp. 292-305, 1963. 